\title{
Die Typhusschutzimpfung in Ostende,
}

\section{das Beispiel der vollständigen Durchimpfung einer größeren Stadt} und ihr günstiges Ergebnis.

Von

\author{
Dr. Fürth und Dr. Pflugbeil, \\ Marine-Stabsärzten \\ und
}

Dr. Oertel, Mar.-Ob.-Ass.-Arzt d. Res.

\section{Allgemeine hygienisehe Verhältnisse und Verbreitung des Typhus in Westflandern.}

Die Sorge für die Gesunderhaltung der Truppen gab sofort nach der Besetzung des flandrischen Küstengebietes Veranlassung, über das Vorkommen von Typhuserkrankungen Erhebungen anzustellen. Diese Aufgabe wurde durch das Fehlen einer Meldepflicht für ansteckende Krankheiten, sowie die Flucht vieler Ärzte sehr erschwert. Die von den zurückgebliebenen Ärzten erhaltenen Angaben erschienen zum Teil wenig glaubwürdig und zuverlässig. So hörte man z. B. in Ostende fast allgemein, Typhuserkrankungen seien dort sehr selten und in den langen Friedensjahren nur vereinzelt beobachtet worden. Dabei mag der Wunsch mitgespielt haben, das bis dahin viel besuchte Seebad nicht in Verruf zu bringen. Jedenfalls erhielten wir schon bei der Durchsicht der Jahrbü̈cher, wie der „Rapports des Commissions médicales provinciales“ und des „Bulletin de-statistique Sanitaire", sowohl von Ostende als auch den übrigen Orten Westflanderns hinsichtlich des Typhus ein anderes Bild. Danach war dieser in den meisten Ortschaften des von uns besetzten Gebietes endemisch. Auch kleinere Epidemien waren zeitweise aufgetreten. Gleichfalls führten die Berichte der Brügger „Commission médicale“ aus den letzten 10 Jahren von den verschiedenen Dörfern und Städten des Küstenlandes manche Todesfälle auf. Die Typhussterbefälle in Brügge und Ostende aus den letrten $\mathbf{5}$ Jahren sind nachstehend zusammengestellt: 


\begin{tabular}{|c|c|c|c|c|c|c|}
\hline & 1909 & 1910 & 1911 & 1912 & 1913 & Summe \\
\hline Brügge & 14 & 5 & 10 & 7 & .16 & 52 \\
\hline Ostende & 5 & 12 & 11 & 5 & 5 & 38 \\
\hline
\end{tabular}

Sie entsprechen ungefähr 1 Promille der Bevölkerung in 5 Jahren.

Als Ursache ist meist das schlechte, durch menschliche und tiorische Schmutzstoffe verunreinigte Genußwasser angegeben. Auch die Einschleppung durch aus Frankreich zurückkehrende Feldarbeiter ist vielfach behauptet worden. In den Berichten wird zwar die Mühe und Sorgfalt anerkannt, die sich der größte Teil der Ärzte bei der Bekämpfung des Typhus gegeben habe. Die jährlichen Erkrankungsziffern zeigen jedoch, wie wenig Erfolg diese Maßnahmen hatten. Bedenkt man, daß trotz großer Bemühung einsichtsvoller Kreise die Meldepflicht für ansteckende Krankheiten in Belgien nicht eingeführt war, so erscheint es berechtigt, die vorliegenden Zahlen der Erkrankungen und Todesfälle an Typhus noch als viel zu niedrig anzunehmen. Aus diesem Grunde wird hier von einer Anführung der Krankheitsziffern aus den jährlichen Berichten abgesehen. Die Verbreitung des Typhus nimmt aber auch bei den mannigfachen sonstigen Mängeln des belgischen Gesundheitswesens nicht wunder. Auf diese Verhältnisse, wie sie sich im Küstengebiet darboten, soll hier nicht näher eingegangen werden. Sie sind von einem von uns bereits früher beleuchtet worden. ${ }^{1}$

Die für Ostende wichtigsten Punkte dieser Art sind: die durchaus mangelhafte Versorgung der Stadt mit Trinkwasser, die Mängel der Wohnverhältnisse der großen Arbeiter- und Fischerbevölkerung und deren geringes Verständnis für die Forderungen der allgemeinen Gesundheitspflege.

Aus allem dem erhellt, daß wir den Typhuserkrankungen unter der bürgerlichen Bevölkerung und der Besserung der hygienischen Verhältnisse von vornherein die größte Aufmerksamkeit schenken mußten, um bei der. unvermeidbaren Berührung unserer Truppen mit den Einwohnern die folgenschwere Ubertragung auf die Besatzungstruppen zu verhindern.

Zunächst galt es, über alle Fälle sofort und zuverlässig unterrichtet zu werden, einmal, um der Ansteckungsgefahr begegnen zu können, dann auch, um über die Verbreitung des Typhus, die sich aus den oben angeführten Gründen nur unvollkommen ermitteln ließ, genaue Kennțis zu erhalten.

1 Fürth, Die Trinkwasserverhältnisse im westflandrischen. Küstengebiet. M. m. W. 1915. Nr. 25.

Derselbe, Hygienische Streiflichter aus Westflandern. Archiv, f. Sch. $u$. Tr.-Hyg. 1916. Bd. XX. 
Es wurde die Meldepflicht für ansteckende Krankheiten eingeführt. Hierbei bedurfte es steter Aufsicht und öfters wiederholter Hinweise, ehe Ärzte und Gemeinden diesem notwendigen Gebot mit der erforderlichen Sorgfalt nachkamen. Bei der Armut und Raumbeschränkung der Häuser in den Landgemeinden und ärmeren Vierteln der Städte bot ein Verbleiben der Kranken in ihren Wohnungen, in denen es auch an Pflege und Behandlung fehlte, die größte Gefahr für den Kranken und seine Umgebung. Es - wurden deshalb in Brügge und Ostende je ein großes Seuchenhospital eingerichtet, und das tberführen eines jeden Typhuskranken dorthin zur Pflicht gemacht. Diese Krankenhäuser bewährten sich sehr gut und wurden von den dort Behandelten und ihren Angehörigen als große Wohltat empfunden. Sie kamen auch vielen Kranken aus der ärmeren Bevölkerung zugute, die wegen Verdachts auf Typhus oder wegen anderer ansteckender Krankheiten in ihnen Aufnahme fanden. $\mathrm{DaB}$ die hier durch genaue klinische und bakteriologische Untersuchung gewährleistete Sicherung der Diagnose die Typhusbekämpfung wirksam unterstiitzte, bedarf kaum besonderer Erwähnung.

In allen Fällen, in denen Verdacht auf Kontaktinfektion im Hause bestand, oder der Betrieb einer Lebensmittelhandlung und Schankwirtschaft daselbst besondere Vorsicht erheischte, wurden alle Hausinsassen auf Keimausscheidung untersucht. Die verseuchten Häuser wurden in Augenschein genommen, als solche durch Anschlag gekennzeichnet, ibr Betreten den Truppen verboten, und die notwendigen Desinfektionen ausgeführt. Bei diesen unterstiitzten die Gemeinden besondere aus unseren Mannschaften gebildete Desinfektionstrupps. Dabei wurde sowohl in diesen Einzelfällen als auch allgemein Reinlichkeit im Hause und in der Umgebung, eine Verbesserung der Wasserversorgung und die Beseitigung der Abfallstoffe angestrebt.

Das waren die zunächst von uns zum Schutze gegen Typhus getroffenen Maßnahmen. Der im Laufe der nächsten Monate immer nachdrücklicher durchgeführte Meldedienst von seiten der Ärzte und Gemeinden, die genaue Eintragung der Fälle in einen Stadtplan, Hausbesichtigungen und Untersuchungen gaben uns nach und nach genaueren Aufschluß über die Ausdehnung des Typhus in Ostende. Wie sich die Fälle in der Stadt vẹrteilten, zeigt der beigefügte Plan. Es ist nicht das Bild, wie es etwa eine Verseuchung der Zentralwasserversorgungsanlage macht, auch gleicht die Verbreitung nicht der durch Milch- oder Gemüsehandel hervorgerufenen Massenerkrankung, vielmehr sieht es mehr nach einer Verbreitung der Seuche von Person zu Person durch Kranke oder Keimträger aus. 
Der weitere Verlauf zeigte leider, dab wir mit den besprochenen Maßnahmen allein den erhofften Abfall der Erkrankungsziffer nicht zu erzielen vermochten. Als die Zahl der Typhusfälle im August 1915 auf 38 stieg, mußten sowohl im Interesse der Stadtbewohner als auch der Besatzungstruppen, unter denen bislang dank der Impfung und der hier leichter durchführbaren sonstigen Vorkehrungen Typhusfälle nur vereinzelt aufgetreten waren, weitere Mittel zu ihrer Bekämpfung erwogen werden. Am wirksamsten erschien die allgemeine Schutzimpfung der Bevölkerung in der Art, wie sie seit Beginn des Krieges bei der gesamten Armee durchgeführt wurde. Dieses Mittel war um so mehr berufen, als sich bei den bisherigen Impfungen deren Gefahrlosigkeit und gute Wirkung gezeigt hatte.

\section{Der Erfolg früherer Typhusschutzimpfungen.}

Typhusschutzimpfungen wurden in größerem Umfang bis jetzt fast ausschließlich bei Truppen durchgeführt. Die von der deutschen Armee bei der südwestafrikanischen Schutztruppe sowie in der britischen, amerikanischen und japanischen Landarmee -und französischen Marine vor dem Kriege gemachten Erfahrungen können als bekannt vorausgesetzt werden.

Uber die Wirkung der Typhusschutzimpfung im deutsehen Heere während des Krieges berichtet Hünermann ${ }^{1}$ ausführlich. Die Typhuskurve des Feldheeres stieg von 0.05 Promille bis Dezember 1914 auf 1.5 Promille, sank dann unter dem Einfluß der Impfung auf 0.015 Promille im März 1915. Ein zweiter geringer Anstieg im Juni-September 1915 wurde durch die Wiederholung der Impfung unterdrückt. Ähnliche Kurven wie das ganze Feldheer weisen die einzelnen Verbände auf. Ungünstige Ergebnisse finden in äußeren Umständen Erklärung. Die Zahl der Todesfälle, die bei den nicht Geimpften 9.6 Prozent beträgt und der Friedenssterblichkeit entspricht, sinkt nach der ersten Einspritzung auf 8. 7 Prozent, nach der zweiten auf 6:6 Prozent, nach der dritten auf 5.3 Prozent, nach der Wiederimpfung bis auf 2.6 Prozent. In den allermeisten Fällen verläuft der Typhus außerordentlich leicht.

Russel ${ }^{2}$ versuchte bereits 1911, eine Typhusepidemie unter der bürgerlichen Bevölkerung einer Stadt durch Sehutzimpfung zu bekämpfen. In Torrington mit 17000 Einwohnern erkrankten im September 227, im

1 Hünermann, Typhusschutzimpfung. Verhandl. d. außerord. Tag. d. D. Kongr. d. i. Med. in Warschau. 1916.

${ }^{2}$ Russel, Journat of the americ. med. ass. Bd. LVIII. Nr. 18. 
Oktober 54 und im November 5 an Typhus, zusammen 286 mit 20 Todesfällen. Bei Beginn der Epidemie wurden 300 bis 400 Leute geimpft. Von diesen erkrankte nur einer und zwar leicht. Von 80 Pflegerinnen ließen sich 45 impfen und waren geschützt. 2 Ungeimpfte bekamen Typhus. Trotz der unvollkommenen Durchführung hatten die Impfungen also einen guten Erfolg.

Uber etwa während des Krieges vorgenommene Schutzimpfungen von städtischen Bevölkerungen konnten wir in der Literatur keine Angaben finden, erhielten auch sonst davon keine Kenntnis.

\section{II. Vorbereitungen zur Impfung.}

Als infolge der zunehmenden Typhuserkrankungen in Ostende und Umgebung Anfang September 1915 vom Korpsarzt die Durchführung der Typhusschutzimpfung in diesem Bezirke angeordnet wurde, war von vornherein anzunehmen, daß die Ergebnisse dieser Maßnahme für die Bewertung des Nutzens der Impfung von besonderer Wichtigkeit sein würden, da hier die Verhältnisse für eine Beurteilung viel günstiger lagen als bei der fechtenden Truppe. Die Truppenstatistiken sind nur unter Berücksichtigung vieler wichtiger Nebenumstände für die einwandfreie Bewertung der Wirkung der Impfung gültig. Örtliche Verhältnisse, Verschiebungen ganzer Verbände und Verschiebungen innerhalb derselben, Neuauffüllungen, die verschiedene Möglichkeit einer exakten Durchführung der Impfung und anderer hygienischer Maßnahmen haben Einfluß auf die Zahlen und machen sie nur bedingt verwertbar.

Diese Nebenumstände kommen weniger in Betracht bei der Schutzimpfung eines geschlossenen Gemeinwesens. In Ostende kamen als begünstigende Umstände noch hinzu, daß infolge des Krieges die Reisemöglichkeit der Bevölkerung fast ganz aufgehoben war, und sich in den hygienischen Verhältnissen infolge der Unmöglichkeit, die durchaus schlechte Wasserversorgung und die Mängel der Wohnverhältnisse zu verbessern, keine durchgreifende Änderung erzielen ließ. Zudem waren wir in der Lage, den genauen Stand der Typhuserkrankungen unter der Einwohnerschaft von etwa 32000 Köpfen in fast einjähriger vorheriger Beobachtungszeit einwandfrei festzustellen.

Außer der städtischen Gemeinde Ostende mit ihren Vororten sollten die Dörfer Steene und Breedene geimpft werden. Diese waren ebenfalls verseucht und mußten als die Hauptbezugsquellen ländlicher Erzeugnisse für Ostende mit in die Impfung einbegriffen werden. 


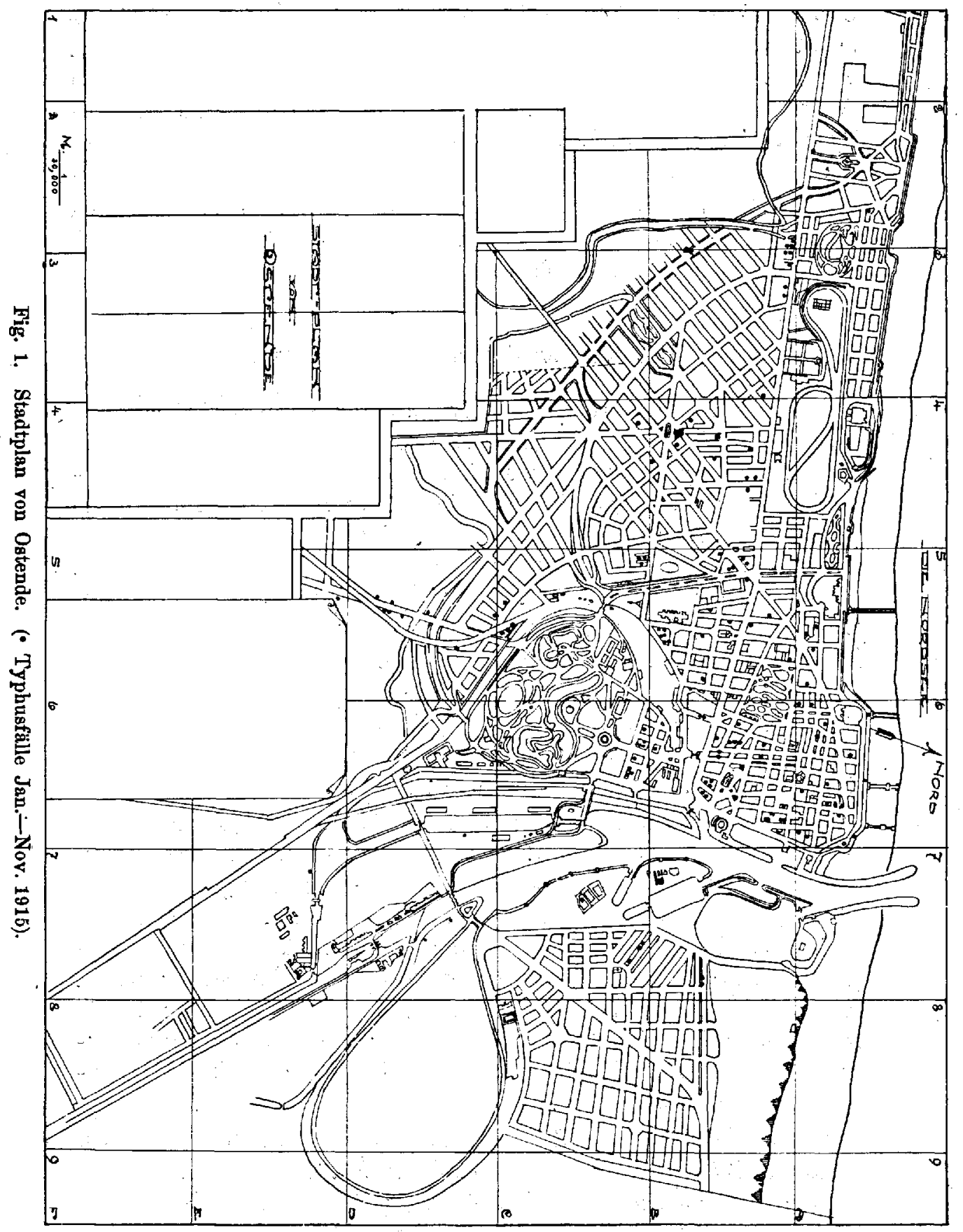


Zunächst galt es, die fremdsprachige Bevölkerüng aufauklären. Zu diesem Zweck belehrte der damalige Korpsarzt persönlich die zusammengerufenen Zivilärzte, die ihrerseits wieder auf ihren Anhang einzuwirken hatten. Es gelang ferner, 14 Helferinnen vom belgischen Roten Kreuz für die Mitarbeit zu gewinnen, die später durch ihre Ausbildung, Sprachenund Ortskenntnis und nicht zuletzt durch ihren Eifer und ihre Geschicklichkeit viel zum Gelingen der Arbeit beigetragen haben. Schließlich wurde die Bevölkerung noch dureh zweisprachlichen Anschlag. und Bekanntmachungen in den Schulen über Wesen, Gefahrlosigkeit, Vorteile und Art der Durchführung der Impfung unterrichtet. Gleichzeitig wurde den Gemeinden befohlen, straßenweise Impflisten anzulegen. Nach Lieferung, Prüfung und Durchzählung dieser Listen ergab sich eine Zahl von etwa 32000 Bewohnern. Um Erfahrungen zu sammeln, wurde schon während dieser Vorbereitungen in einem Vororte von etwa 700 Einwohnern, wo der. Typhus besonders heftig aufgetreten war, die Typhusschutzimpfung durchgeführt. Die Schwierigkeiten waren gering und der Erfolg ermutigend. $\mathrm{Zu}$ den weiteren Vorbereitungen gehörte die Aufstellung von 5 bis 7 Impfkolonnen bestehend aus: .

1 deutschen Arzt,

2 Helferinnen (zur Listenführung),

1 Sanitätsunteroffizier,

1 bis 2 belgischen Schutzleuten.

Das gesamte Impfgebiet wurde in 3 Bezirke eingeteilt, die nacheinander durchgeimpft werden sollten, weil die dreimalige Durchimpfung der gesamten Einwohnerzahl im Abstand von 7 bis 8 Tagen unmöglich erschien.

Mehrere Straßen mit zusammen 300 bis 700 Einwohnern, je nach den örtlichen Schwierigkeiten, wurden zu einer Untergruppe, entsprechend der Tagesleistung einer Impfkolonne zusammengefaßt, mehrere Untergruppen zu einer Gruppe, entsprechend der Tagesleistung aller angesetzten Kolonnen. Dabei ergab sich folgendes Bild:

I. Bezirk: Ostende innere Stadt, 8 Gruppen $=40$ Untergruppen $=$ rund 20000 Einwohner.

II. Bezirk: Ostende Vororte, 3 Gruppen $\doteq 15$ Untergruppen $=$ rund 7500 Einwohner.

III. Bezirk: Ländliche Gemeinden, 3 Gruppen $=11$ Untergruppen $=$ rund 6500 Einwohner. 


\section{Ausführung der Impfung.}

Der Impfung wurden unterzogen alle Personen vom 3. bis 60. Lebensjahr, wobei an den Grenzen frühzeitig gealterte bzw. wenig entwickelte Personen befreit blieben. Außer infolge Alters waren befreit: Schwerkranke (besonders Tuberkulöse, Nierenkranke), Schwangere und Personen, die 1914/15 nachweișlich Typhus überstanden hatten.

Die Impfung wurde ausgeführt nach den bei uns geltenden Vorschriften: dreimalige subkutane Impfung mit 0.5 bis $1.0 \mathrm{ccm}$ Impfstoff im Abstand von 7 bis 10 Tagen. Impfstelle an der Brust, seltener am Rücken. Der Impfstoff war polyvalenter Typhússchutzimpfstoff von verschiedenen deutschen Instituten und vom Chef des Feldsanitätswesens zur Verfügung gestellt. Bei Kindern unter 6 Jahren wurde mit folgenden Mengen geimpft:

\begin{tabular}{cc||c|c|c}
\hline & & $\begin{array}{c}\text { 1. Impfung } \\
\text { ccm }\end{array}$ & $\begin{array}{c}\text { 2. Impfung } \\
\text { ccm }\end{array}$ & $\begin{array}{c}\text { 3. Impfung } \\
\text { ccm }\end{array}$ \\
\hline \hline Im 3. Lebensjahr. . . & 0.25 & 0.5 & 1.0 \\
"4. & 4. . & 0.3 & 0.6 & 1.0 \\
5. & . . . & 0.4 & 0.8 & 1.0
\end{tabular}

Die Impfung fand straßenweise nachmittags von 3 bis 6 Uhr statt. Als Impiraum wurden geeignete Privathäuser, Wirtschaften oder Schulen gewählt. Impfraum und Impfzeit wurden tags zuvor den beteiligten Personen durch die Stadtpolizei mitgeteilt.

Die erste dreimalige Durehimpfung fand statt vom 4 . Oktober bis 10. Dezember 1915. Naç ungefähr 1/2 Jahr wurde wie in anderen Fällen die Impfung wiederholt, eine zweimalige Impfung von 0.5 und $1.0 \mathrm{ccm}$ unter sonst gleichen Bedingungen vom 14. Juli bis 1. August 1916. Nachstehend folgt eine Übersicht über die vorgenommenen Impfungen:

\begin{tabular}{l||c|c|c|c|c|c}
\hline \hline & $\begin{array}{c}\text { Be- } \\
\text { wohner }\end{array}$ & Geimpft & $\begin{array}{c}\text { Nicht } \\
\text { geimpft }\end{array}$ & $\begin{array}{c}\text { Wegen } \\
\text { Alters }\end{array}$ & $\begin{array}{c}\text { Wegen } \\
\text { Krank- } \\
\text { beit }\end{array}$ & $\begin{array}{c}\text { Wegen } \\
\text { anderer } \\
\text { Grüade }\end{array}$ \\
\hline \hline 1. Impfung 1915 & $\mathbf{3 2 8 1 1}$ & 27472 & 5339 & 4212 & 772 & 355 \\
2. $"$ 1916 & 32728 & 27008 & 5720 & 4471 & 886 & 363
\end{tabular}

\section{Aüßer der Impfung getroffene Maßnahmen gegen Typhus.}

Der in Ostende verbreiteten Ansicht, daß die durchaus mangelhafte und unhygienische allgemeine Wasserversorgung der Stadt an der Verbreitung des Typhus die Hauptschuld trage, konnten wir uns nicht anschließen. Schon das üble Aussehen und der stark salzige. Geschmack des 
Wassers mußte die Menschen abhalten, es für Genußzwecke zu verwenden. Viele der verseuchten Häuser waren zudem nicht an das Leitungsnetz angeschlossen. 'Sehließlich hätte dessen Verseuchung zu einer größeren Epidemie und zu plötzlichen Explosionen führen müssen. Das hielt uns naturgemäß nicht davon $a b$, alles für Verbesserung der Trinkwasserverhältnisse Ostendes.Dienliche zu versuchen. Doch ist es bei der Schwierigkeit der Verhältnisse bis heute noch nicht einmal gelungen, so weit eine Besserung des dem städtischen Wasserwerk allein zur Verfügung stehenden Binnenkanalwassers zu bewirken, daß mit Hilfe eines der gebräuchlichen ehemischen Reinigungsverfahren ein brauchbares Genußwasser sich hätte gewinnen lassen. Alle in dieser Richtung unternommenen Versuche, zuletzt die Chlorung des Wassers, blieben infolge des überaus hohen Gehaltes an Salz und oxydablen Stoffen erfolglos. Auch der Einzelversorgung der Häuser durch Grundwasserbrunnen und Regenwasserzisternen wurde stete Aufmerksamkeit geschenkt, wo es nottat, Brunnen geschlossen, und die Bevölkerung zu einer regelmäßigen Reinigung der Regenwasserbehälter angehalten.

Die Verbreitungsart des Typhus sprach, wie bereits erwähnt, mehr für eine Ansteckung yon Person zu Person, sei es durch Kranke, Genesende oder gesunde Keimausscheider. Deshalb waren wir bestrebt, soweit es die uns zur Verfügung stehenden Hilfskräfte und Mittel erlaubten, unter den früher Erkrankten und ihren Hausgenossen auf Keimträger zu fahnden. Gleichwohl erschien eine Ubertragung durch Milch nicht ausgeschlossen. Daher wurde die Untersuchung auf alle Milchhändler, ihre Familien und Bedienstete ausgedehnt. Es kamen vor allem die Kleinbauern der Außenviertel Ostendes in Betracht, die, vielfach nur im Besitze einer Kuh, unter der Hand Milch verkauften und einer amtlichen Prüfung nicht unterliegen.

Auch diese Untersuchung auf Keimträger wurde wie die Impfung durch Ausarbeitung eines zweckmäßigen Arbeitsplanes sehr erlejchtert. Es ist wohl von allgemeinem Interesse, diesen hier kurz zu beschreiben. Für jedes Haus, dessen Insassen aus einem der vorgenannten Gründe untersucht werden sollten, wurde eine Hausliste angelegt, wie sie nachfolgend abgedruckt ist.

Die Gemeinde hatte Namen und Personalien der Hausbewohner einzutragen. Einer von uns suchte dann die Familie in Begleitung einer freiwilligen Helferin persönlich auf und zog die erforderlichen Erkundigungen ein. Dieses Hausblatt, mit Zeichen und Nummer versehen, bildete eine dauernde' Unterlage für weitere Feststellungen und spätere Nachuntersuchungen. Im Laboratorium wurde auf Grund dieser Liste die Utbersendung des Untersuchungsmaterials dergestalt vorbereitet, daß für jedes Haus ein Schein ausgestellt wurde, der die Namen aller zu Untersuchenden 
Fürth, Pflugbeil und Oertel:

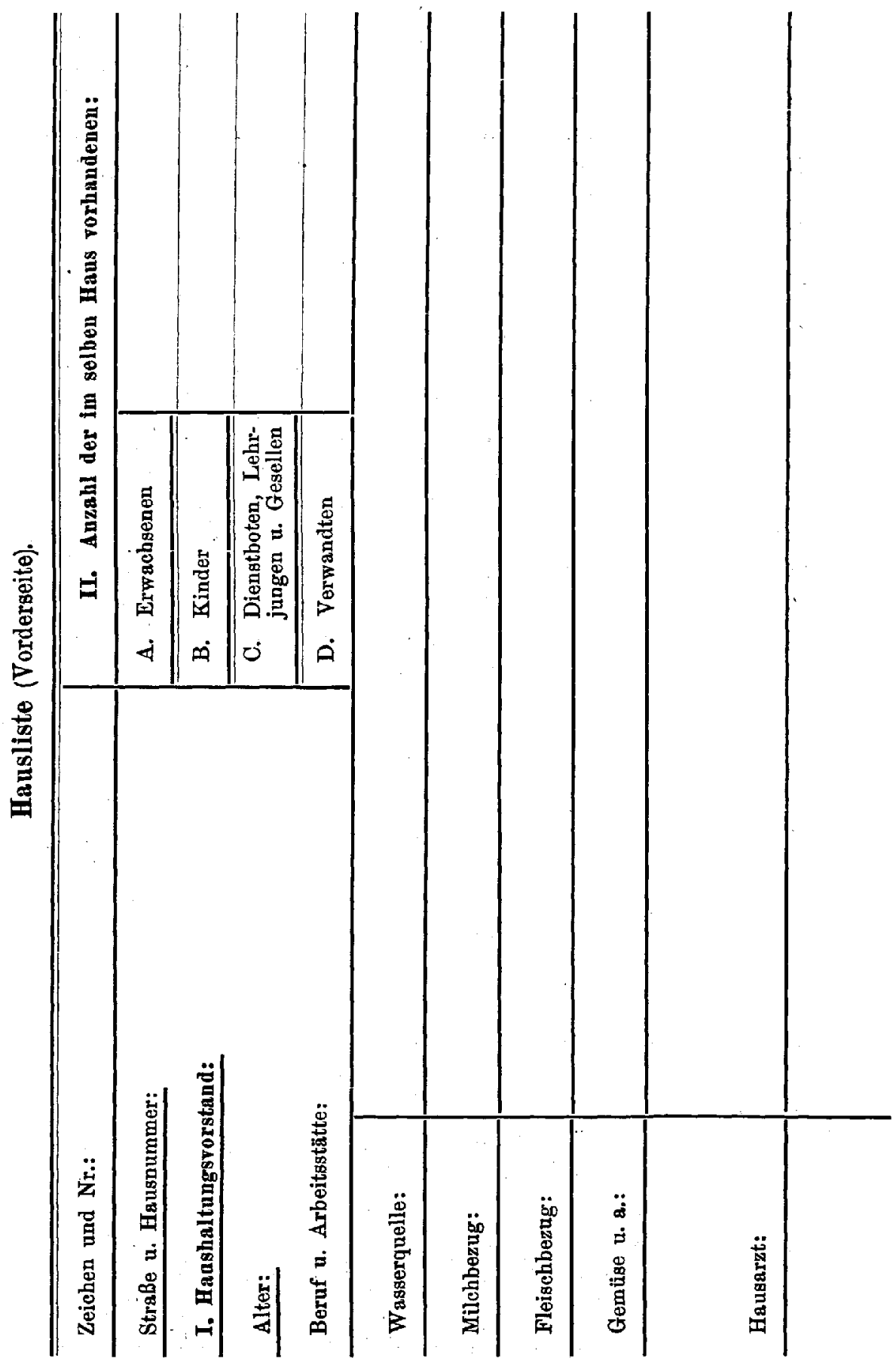


Die Typhusschutzimpfung in Ostende.

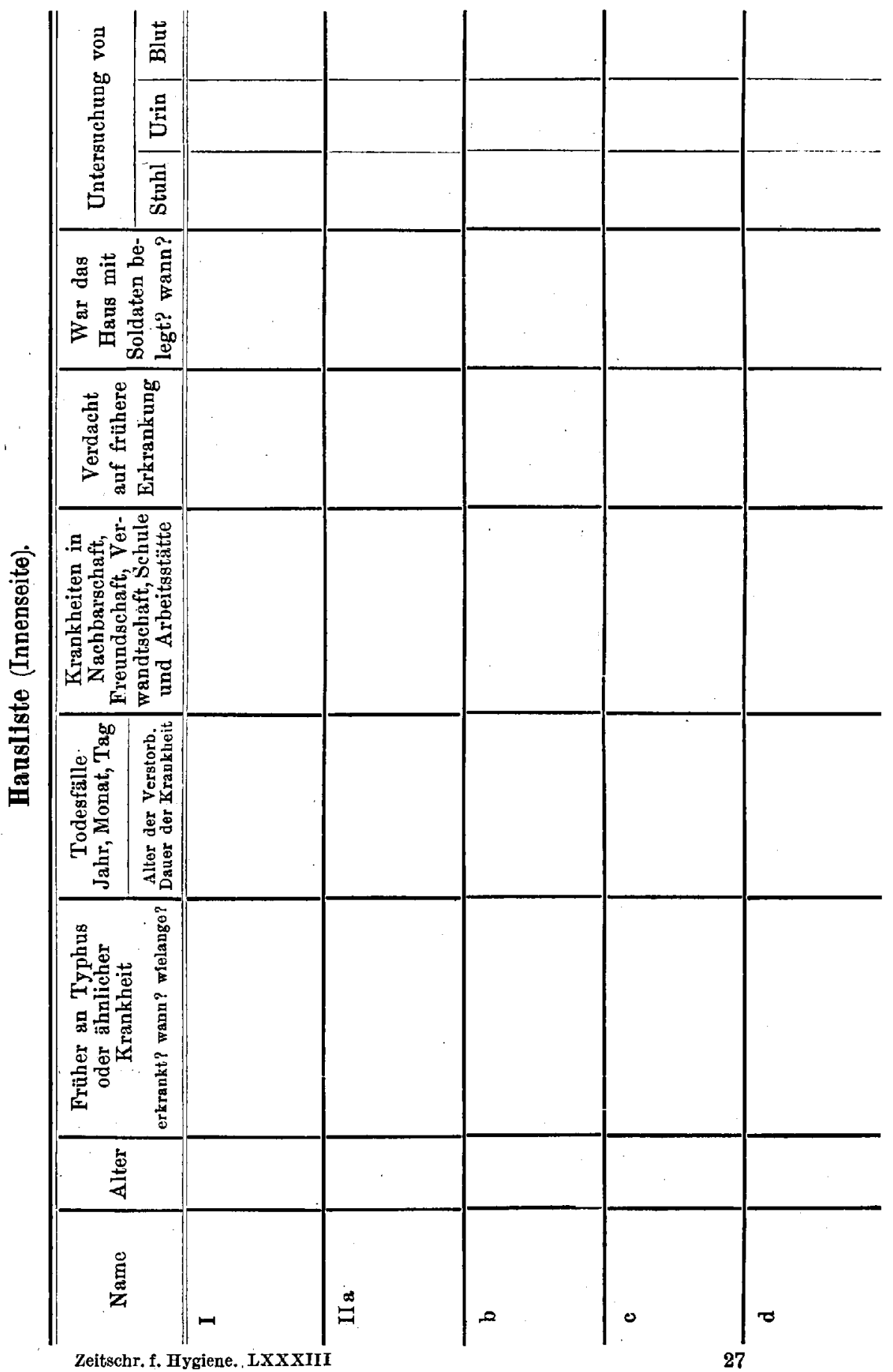


und eine genaue Anweisung für die Lieferung von Material enthielt. Zugleich mit diesem Schein wurde die nötige Anzahl von Versandgefäßen, fertig mit Name und Nummer bezeichnet, den Hausbewohnern übergeben, die zum bestimmten Zeitpunkt selbst die Proben dem Laboratorium einzuliefern hatten. Dort genügte bei der Verarbeitung dann Zeichen und Nummer zur Kennzeichnung, was die Massenuntersuchungen sehr vereinfachte. Die Hauslisten blieben mit einem Vermerk über das Untersuchungsergebnis im Laboratorium aufbewahrt.

Die Verteilung und das Ergebnis der Untersuchungen von Stuhl und Urinproben von insgesamt 1466 so untersuchten Personen ist in folgender Liste zusammengestellt:

\begin{tabular}{|c|c|c|c|c|c|}
\hline Untersuchte & Ostende & Steene & Breedene & $\begin{array}{l}\text { Zand- } \\
\text { voorde }\end{array}$ & $\begin{array}{l}\text { Alle } \\
4 \text { Orte }\end{array}$ \\
\hline Aus verseuchten Hänsern & 624 & 163 & 62 & 26 & 875 \\
\hline Aus Milchbezugsquellen . & 185 & 209 & 160 & 37 & 591 \\
\hline Gesamtzahl: . ... . & 809 & 372 & 222 & 63 & 1466 \\
\hline \multicolumn{6}{|l|}{ Keimträger: } \\
\hline Typhus . . . . . . & 4 & 4 & 1 & - & 9 \\
\hline Paratyphus . . . . . . & 3 & - & - & - & 3 \\
\hline
\end{tabular}

Der sicherste Weg, Ansteckung von seiten der Keimträger zu vermeiden, wäre gewesen, diese dauernd in einem Krankenhause abzusondern. Doch versuchten wir zunächst mit Rücksicht darauf, daß so Männer und Frauen ihrer Familie entzogen wurden, einen anderen Weg. Wir nahmen zunächst die als Keimträger erkannten Personen - Kinder mit einer erwachsenen verwandten Person - zu einer genauen Nachuntersuchung ins Krankenhaus auf und ließen ihnen hier bis zum Abschluß der Untersuchung, die 3 bis 8 Tage dauerte, eine eingehende Belehrung über die von ihrer Seite den Mitmenschen drohende Gefahr zuteil werden. Zugleich unterwiesen wir sie in den nötigen Desinfektionsmaßnahmen. Bei der Entlassung erhielten sie ein besonders für sie in deutscher und flämischer Sprache gedrucktes Merkblatt, das ihnen dauernd das für sie Wissenswerte vor Augen halten sollte; auch enthielt es eine Anleitung zur $\mathrm{Be}-$ reitung von Desinfektionslösungen. Der Wortlaut war folgender:

\section{Vorschrift für Typhusbazillenträger.}

Name:

Wohnung:

Ausgehändigt am: 
Wie die Untersuchung ergeben hat, scheiden Sie mit den täglichen Entleerungen (Stuhl und Urin) Stoffe aus, die den Typhus auf andere Menschen übertragen.

Diese Utbertragung kann geschehen:

1. unmittelbar durch den Kot und Urin;

2. durch Gegenstände, die mit Kot und Urin boschmutzt sind, besonders Thre Hände und Thre Wäsche.

Die Stoffe, die die Krankheit übertragen, werden durch die Desinfektion vernichtet und unschädlich gemacht.

Es ist daher für Sie Pflicht, jede Kot- und Urinentleerung sowie die Hände und die Wäsche zu desinfizieren.

Wenn Kot oder Urin in ein Gefäß entleert wird, so ist er in diesem Gefäß sofort mit der gleichen Menge Kalkmilch zu übergießen. Die Mischung muß durchgemischt werden und vor dem Ausgießen 1 Stunde stehen. In die von Ihnen benutzte Abortgrube oder Urinrinne wuß täglich $1 / 2$ Liter Kalkmilch eingegossen werden.

Nach jeder Stuhl- und Urinentleerung müssen Sie die Hände gründlich in einer 5 prozentigen Lösung von Kresolseifenlösung waschen.

Die getragene $W a ̈ s c h e ~ m u ß$ vor dem allgemeinen Waschen 1 Stunde in 5prozentige Kresolseifenlösung gelegt werden.

Jede in Threr Umgebung vorgekommene fieberhafte Erkrankung ist verdächtig und muß sofort dem Arzt (oder der Behörde) gemeldet werden, wobei diese Vorschrift vorzuzeigen ist.

Wenn Sie diese Vorschriften, deren Befolgung dauernd streng kontrolliert wird, nicht, beachten, müssen Sie für dauernd in ein Krankenhaus gebracht werden.

Es folgt eine Anweisung zur Bereitung der Desinfektionslösungen.

Mit dem Stempel der Ortskommandantur versehen, diente dieses Merkblatt zugleich als Ausweis beim Bezug von Desinfektionsmitteln, zu deren kostenloser Verabfolgung die Gemeinden angehalten wurden.

Da noch die Wohnung der Keimträger durch Anschlag gekennzeichnet und auf die durch ihr Betreten bedingte Gefahr aufmerksam gemacht wurde, auch die Garnisonärzte Befehl erhielten, sich wöchentlich einmal durch Besuch der Häuser von der ordnungsgemäßen Befolgung der Anordnungen $\mathrm{zu}$ überzeugen, glaubten wir genügend vorgesorgt zu haben. Leider mußten wir späterhin doch auf die strengere Maßnahme der Isolierung im Krankenhaus zurückkommen, als trotz mehrfacher Warnungen die Vorschriften zum Teil gar nicht oder mangelhaft befolgt wurden, und mit Sicherheit zwei Typhusfälle auf Ansteckung durch einen Keimträger zurückgeführt werden konnten. 
Von den 12 Keimausscheidern, die monatlich einmal nachuntersucht wurden, blieben in der Folgezeit vier Typhusdauerausscheider. Sie fanden im Ostender Seuchenhospital dauernd Aufnahme. Hierdurch wurden die Aufgaben des Typhuskrankenhauses um eine wichtige vermehrt. Besonders wertvolle Dienste leistete es jedoch für die Beurteilung des Erfolges der Schutzimpfung in der auf diese folgenden Zeit. Kam es doch vor allem darauf an, jeden irgendwie auf. Typhus verdächtigen Fall klinisch und bakteriologisch genau zu untersuchen und zu klären. Damit kommen wir zum näehsten Abschnitt unserer Mitteilungen.

\section{Ergebnis der Impfung.}

Die Haltung der Bevölkerung war bei der ersten Durchimpfung anfangs etwas ängstlich, später aber, als sie die Harmlosigkeit und den Nutzen eingesehen hatte, sehr entgegenkommend und willig. Trotzdem empfiehlt es sich, bei der Impfung einer zahlreichen Zivilbevölkerung langsam und schrittweise vorzugehen, weil man von ihrem guten Willen und Ordnungssinn sehr abhängig ist. Da einerseits die Impfungen in regelmäßigen $\mathrm{Ab}$ ständen von 7 bis. 10 Tagen vorgenommen werden müssen, andererseits aber mit Zuspätkommen, entschuldigtem und unentschuldigtem Fernbleiben, sowie mit unerwartet eintretenden Krankheiten gerechnet werden muß, so kann man leicht die Utbersicht verlieren, wenn man nicht rechtzeitig Maßnahmen dagegen ergreift. So müssen insbesondere von vornherein Nachimpfungstermine, die neben den Hauptimpfungen herlaufen, vorgesehen sein.

Gesundheitliche Schädigungen durch die Impfungen wurden in keinem Falle beobachtet, dagegen war der Nutzen in die Augen springend. Für die ausgezeichnete Wirkung der Schutzimpfung sprechen besonders zwei Tatsachen:

1. Vor der Impfung trat der Typhus wahllos hier und da im gesamten Gebiet.auf. Er verschwand mit der Impfung fortschreitend in den nacheinander durchgeimpften Bezirken vollständig, erfuhr aber in den noch nicht geimpften keine Verminderung. Mit der Beendigung der Impfung hörte er auf.

2. Es. wurden im fraglichen Gebiet beobachtet:

\begin{tabular}{l||c|c}
\hline \hline & Typhusfälle & Todesfälle \\
\hline \hline Vor der Impfung Dezember 1914 bis November 1915 & 213 & 18 \\
Nach der Impfung Dezember 1915 bis November 1916 & 6 & 0
\end{tabular}

Von den 6 an Typhus erkrankten Personen sind 4 nicht geimpft, 2 wegen Fehlens in den Listen, 2 wegen Alters (60 und 65 Jahre). Die 
beiden trotz der Typhusschutzimpfung erkrankten Personen lebten zusammen mit einer Bazillenträgerin. Diese hatte es verstanden, bei der regelmäßigen Untersuchung statt ihres eigenen fremdes Material untersuchen zu lassen; nach der Erkrankung ihrer Angehörigen wurde sie im Seuchenhospital isoliert.

Außer den erwähnten 6 Typhuskranken wurden in der Zeit vom Dezember 1915 bis November 191612 Typhusverdächtige in das Seuchenkrankenhaus eingeliefert, bei denen einwandfrei folgende Krankheiten gefunden wurden:

1. Septischer Abort, $f$.

2. Krupöse Pneumonie.

3. Desgleichen.

4. Appendizitis, $\dagger$ (Sektion).

5. Otitis media, Meningitis, $\dagger$ (Sektion):

6. Krupöse Pnẹumonie.

7. Tuberkulöse Meningitis, $\dagger$ (Sektion).

8. Leichter Darmkatarrh.

9. Kein krankhafter Befund.

10. Leichter Darmkatarrh.

11. Miliartuberkulose, $\dagger$ (Sektion).

12. Leichter Darmkatarrh.

Es sei noch angeführt, $d a ß$ im gleichen Zeitraum des Vorjahres nur bei 8 mit Typhusverdacht eingelieferten Personen sich dieser nicht bestätigte, und nach der Impfung neben den erwähnten 12 Fällen noch mehrere andere poliklinisch im Zusammenwirken mit den belgischen Ärzten von uns geklärt und dadurch nicht im Seuchenhospital aufgenommen wurden.

\section{Der Typhus im geimpften 0stende und dem nicht geimpften Nachbargebiete.}

Für die Beurteilung der Wirkung der Typhusschutzimpfung auf den Verlauf des Typhus in Ostende war aber weiterhin von Wert, daß wir durch Aufzeichnung der in den Nachbargemeinden Ostendes und der weiteren. Umgebung in demselben Zeitraum auftretenden Typhusfälle in der Lage waren, eine geimpfte Stadt ihrer nicht durchgeimpften Nachbarschaft gegenüberzustellen. In der beigefügten Kurve finden sich neben den Fällen Ostendes 1. die Zahlen von Brügge, 2. die des von unseren Truppen besetzten Küstengebietes einschließlich Brügges, 3. die in dem Brügger Seuchenhospital behandelten Fälle, die sowohl aus unserem Gebiet als auch dem benachbarter Korps stammen und dadurch über den Verlauf 
Fürth, Pflugbeil und Oertel:

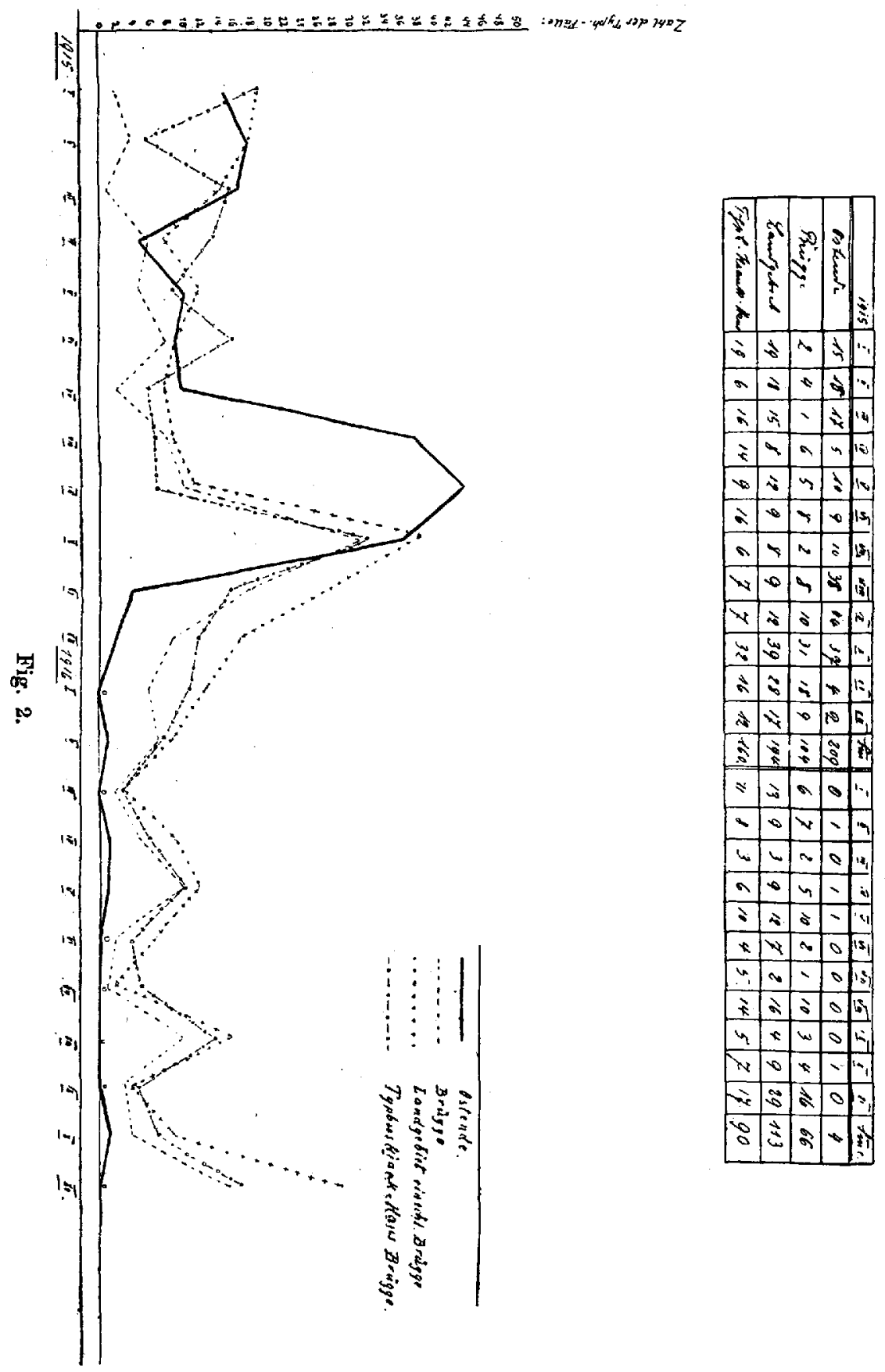




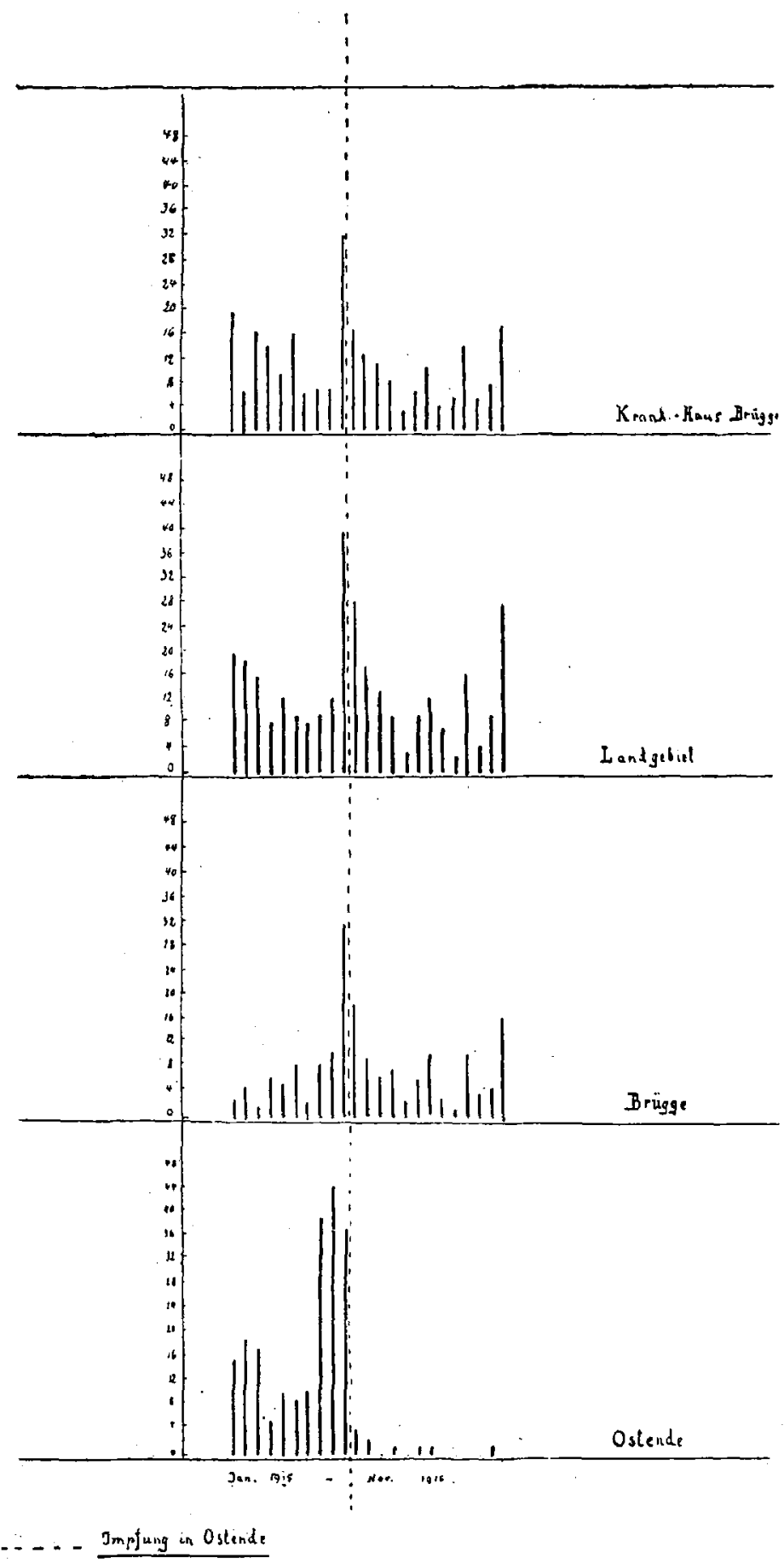

Fig. 3. 
der Erkrankungen im weiteren. Umkreis ein Bild zu geben vermögen. Wahrscheinlich sind die Zahlen aus dem Landgebiet noch zu niedrig, da bei der verhältnismäßig schlechten ärztlichen Versorgung der verstreut liegenden kleinen Gemeinden eher leichte Fälle, namentlich bei Kindern, unbehandelt blieben als in den Städten, in denen zudem für die ärmere Bevölkerung poliklinische Sprechstunden und Behandlung durch besondere Armenärzte eingerichtet waren.

In den Ortschaften des von uns besetzten Gebietes waren von vornherein zur Verbesserung der hygienisehen Verhältnisse die gleichen Mabnahmen getroffen wie in Ostende. Vor allem trat auch hier sofort eine strengere therwachung des Gésundheitszustandes der bürgerlichen Bevölkerung ein, die ermöglichte, verdächtige Fälle frühzeitig ihrer Umgebung zu entziehen und in geeignete Pflege zu bringen. Auch die sonstigen Maßnahmen waren in jedem Einzelfalle von Typhus die gleichen.

Weiterhin boten sich auch insofern dieselben für einen einwandfreien Vergleich günstigen Verhältnisse, als neue Einschleppungen an einen Ort durch infizierte Zureisende oder durch auf der Reise erfolgte Ansteckung Einheimischer nicht möglich waren, da infolge der Lage der Ortschaften in der Nähe des Kampfgebietes ein allgemeines Reiseverbot für die bürgerliche Bevölkerung bestand.

Aus allem ersehen wir, daß die besonderen Umstände für eine sichere Beurteilung der Impfwirkung günstig waren.

Bei einem Vergleich der Kurven bemerken wir zunächst, daß die jahreszeitlichen Schwankungen an allen Orten ziemlich übereinstimmen. Auch ist überall eine Steigerung der Typhusfälle im Herbst zu erkennen. Letztere Beobachtung ist beim Typhus nichts Seltenes. Für die hiesigen Verhältnisse gelten wohl die auch sonst beschuldigten Ursachen, wie z. B. die im Herbst gesteigerte Empfänglichkeit für Krankheiten des Darmes, stärkerer Gehalt des Bodens an infektiösen Keimen, vermehrte Feldarbeit u. a. m. Die Schwankung in der Typhusfrequenz hier mit dem Steigen und Sinken des Grundwasserspiegels in Zusammenhang zu bringen, dürfte in einem Lande mit örtlich so verschiedenen und wechselnden Grundwasserverhältnissen auf noch größere Schwierigkeit stoßen als anderwärts.

Weiterhin zeigen unsere Kurven allerorts eine Verminderung der Typhusfälle im zweiten Beobachtungsjahre. Man wird uns ohne weiteres beipflichten, wenn wir hier die für die Besserung der hygienischen. Verhältnisse getroffene Mühewaltung und die bei den vorkommenden Typhusfällen angewandten besonderen Maßnahmen an erster Stelle zur Erklärung heranziehen. Aber auch die viel beobachtete Schwankung der Typhusfrequenz an einem Ort kann diese Tatsache mit erklären. Gegen den etwa 
erhobenen Einwand, die mannigfachen Verordnungen, vor allem die Furcht vor Überführung der Kranken in ein Krankenhaus und vor Bezeichnung der verseuchten Häuser hätten zur Verheimlichung der Fälle geführt, und dies gäbe die Erklärung für den Abfall der Kurve, führen wir folgendes an: Die arme Bevölkerung war im Gegenteil froh, in der schweren Kriegszeit, in der sich die ärztliche Versorgung gleich der ganzen Lebenshaltung sehr verschlechtert hatte, der Sorge für ihre Kranken enthoben zu werden, um so mehr, als sie diese in den gut geleiteten Hospitälern unter der Pflege von Ordensschwestern gut und kostenfrei versorgt wußte. Aúch war an den meisten Orten die Berührung mit den Besatzungstruppen eine zu große, als daß langdauernde schwere Krankheitsfälle hätten verheimlicht werden können, deren Anzeige den Familien, der Gemeinde und dem Arzt bei Strafe zur Pflicht gemacht war. Besonders trifft dies auch für die größeren Städte, wie Ostende und Brügge, zu. Daß den Todesfällen gleichfalls die nötige Beachtung geschenkt wurde, ist selbstverständlich.

Es sei auch darauf hingewiesen, daß bei Durchführung der Typhusschutzimpfung in Ostende der veränderte Verlauf des Typhus nach einer Schutzimpfung bereits bekannt war, wir deshalb von vornherein auch jeder leichter fieberhaft verlaufenden Krankheit Beachtung schenkten. Gerade von seiten der belgischen Ärzte konnten wir nach der Impfung einer größeren Aufmerksamkeit allen irgendwie verdächtigen Fällen gegenüber sicher sein, da die durchgreifende Maßnahme gerade in ärztlichen Kreisen das regste Interesse geweckt und zur Kritik angeregt hatte.

Nun zeigt aber einerseits der Typhus auch nach der Impfung in der Regel noch einen derartigen Verlauf, daß eine Erkrankung bei einiger Sorgfalt nicht so leicht übersehen werden kann, andererseits kamen im Typhuskrankenhaus in Ostende in der Folgezeit, wie bereits oben festgestellt, mehr Fälle von Typhusverdacht ohne vorliegende Typhuserkrankung in Zugang als im Vorjahre. Daraus kann man mit Recht folgern, daß ein absichtliches oder fahrlässiges Verschweigen von Typhus sich höchstens auf ganz vereinzelte leichte Fälle beschränkt hat.

\section{Schlußbemerkungen.}

Fassen wir zum Schluß die hier mitgeteilten Erfahrungen zusammen, so glauben wir berechtigt zu sein, die Typhusschutzimpfung der bürgerlichen Bevölkerung Ostendes als einen vollen Erfolg und einen gelungenen Versuch zu bezeichnen, den Wert der Typhusschutzimpfung einwandfrei - zunächst für die Dauer eines Jahres - darzutun. 
Die Mängel, die der Verwertung des ungeheuren, von der Armee gelieferten Materials für die sichere Beurteilung letzterer Frage anhaften, fallen in unserem Falle weg; dort handelt es sich stets um eine Summe von Einzelerfahrungen, die verschiedene Beobachter in den verschiedensten Gegenden, unter den mannigfaltigsten Ansteckungsmöglichkeiten, den wechselndsten Lebens-, Wohnungs- und Ernährungsbedingungen machten. Dem späteren Beurteiler wird es schwer, wenn nicht unmöglich sein, ohne Kenntnis der jeweiligen Verhältnisse aus den sicher erheblich voneinander abweichenden Berichten das rechte Schlußurteil zu fällen.

In Ostende konnten dagegen' ein und dieselben Beobachter die Impfung einer größeren Stadt unter gleich bleibenden Verhältnissen vorbereiten, durchführen' und unter den gleichen Bedingungen die Wirkung ein Jahr lang verfolgen. Die gleichzeitige genaue Feststellung des Typhusverlaufs in der näheren und weiteren Umgebung, woselbst die Impfung nicht erfolgt war, machte die Beurteilung besonders wertvoll.

Bei der Wertung des auffallend guten Erfolges darf man allerdings einen Umstand nicht unbeachtet lassen, dem dabei wahrscheinlich einige Bedeutung zukommt. Infolge des durch den Krieg verursachten Darniederliegens von Handel und Verkehr und der hierdurch bestehenden Arbeitslosigkeit eines großen Teiles der Bevölkerung wurden ganz andere Bedingungen für die Verbreitung von Seuchen geschaffen, als die sind, unter denen eine schwer arbeitende oder beweglichere Bevölkerung, sowie der den Anstrengungen und Unbilden des Krieges ausgesetzte Soldat steht, der zudem viel seinen Aufenthaltsort wechselt. Erschöpfende Arbeit und angestrengte Tätigkeit schaffen eine erhöhte Disposition für Infektionskrankheiten, und ein gesteigerter Reiseverkehr ist stets von Einfluß auf die Verbreitung von Seuchen gewesen.

Mögen diese Umstände auch zu dem überraschend guten Erfolg mit beigetragen haben, der sorgfältig durchgeführten Schutzimpfung gebührt jedoch der Hauptverdienst dafür, daß der Typhus heute in Ostende kaum noch auftritt. Das Beispiel Ostendes zeigt danit, daß die planmäßig durchgefïhrte Typhusschutzimpfung wohl das erfolgreichste Mittel zum Kampfe gegen die Seuche darstellt. 